\title{
Distribution of Active and Resting Periods in the Motor Activity of Patients with Depression and Schizophrenia
}

\author{
Ole Bernt Fasmer ${ }^{1,2,3}$, Erik Hauge ${ }^{4}$, Jan Øystein Berle ${ }^{1}$, Steven Dilsaver ${ }^{5}$, and Ketil J. Oedegaard ${ }^{1,2,3}$ \\ 1 Division of Psychiatry, Haukeland University Hospital, Bergen, Norway \\ ${ }^{2}$ Department of Clinical Medicine, Section for Psychiatry, University of Bergen, Bergen, Norway \\ ${ }^{3}$ K.G. Jebsen Centre for Research on Neuropsychiatric Disorders, Bergen, Norway \\ ${ }^{4}$ Olaviken Psychiatric Hospital, Bergen, Norway \\ ${ }^{5}$ Comprehensive Doctors Medical Group, Arcadia, CA, USA
}

\begin{abstract}
Objective Alterations of activity are prominent features of the major functional psychiatric disorders. Motor activity patterns are characterized by bursts of activity separated by periods with inactivity. The purpose of the present study has been to analyze such active and inactive periods in patients with depression and schizophrenia.

Methods Actigraph registrations for 12 days from 24 patients with schizophrenia, 23 with depression and 29 healthy controls.

Results Patients with schizophrenia and depression have distinctly different profiles with regard to the characterization and distribution of active and inactive periods. The mean duration of active periods is lowest in the depressed patients, and the duration of inactive periods is highest in the patients with schizophrenia. For active periods the cumulative probability distribution, using lengths from 1 to $35 \mathrm{~min}$, follows a straight line on a log-log plot, suggestive of a power law function, and a similar relationship is found for inactive periods, using lengths from 1 to $20 \mathrm{~min}$. For both active and inactive periods the scaling exponent is higher in the depressed compared to the schizophrenic patients.

Conclusion The present findings add to previously published results, with other mathematical methods, suggesting there are important differences in control systems regulating motor behavior in these two major groups of psychiatric disorders.
\end{abstract}

Psychiatry Investig 2016;13(1):112-120

Key Words Schizophrenia, Depression, Actigraphy, Motor activity.

\section{INTRODUCTION}

Schizophrenia and major depressive disorders are both major health problems in all parts of the world, ${ }^{1,2}$ and are, in common with other functional psychiatric disorders, defined by their clinical features. Finding objective, biological, differences between these disorders could give important insights in relation to pathophysiology and classification. Electroencephalography is one commonly used method for this purpose, ${ }^{3}$ another is recording of motor activity. Alterations of motor activity

Received: September 5, 2014 Revised: February 16, 2015

Accepted: March 28, 2015 Available online: October 13, 2015

$\triangle$ Correspondence: Ole Bernt Fasmer, MD, PhD

Department of Clinical Medicine, Section for Psychiatry, University of Bergen, Haukeland University Hospital, Bergen N-5021, Norway

Tel: +47 41550327, Fax: +47 55958436, E-mail: ole.fasmer@k1.uib.no

(a) This is an Open Access article distributed under the terms of the Creative Commons Attribution Non-Commercial License (http://creativecommons.org/licenses/by$\mathrm{nc} / 3.0$ ) which permits unrestricted non-commercial use, distribution, and reproduction in any medium, provided the original work is properly cited. are prominent features in several psychiatric disorders, such as $\mathrm{ADHD},{ }^{4}$ mood disorders and schizophrenia, ${ }^{5,6}$ with the most pronounced disturbances seen in the catatonia. ${ }^{7}$ In a number of studies of patients with psychiatric disorders objective registrations of motor activity have demonstrated differences in activity patterns compared to normal controls and between disorders. ${ }^{8-11}$ However, assessment of such changes has only to a limited extent been implemented in routine clinical practice.

We have in previous studies, using actigraph registrations combined with mathematical analyses of variability and complexity, shown that patients with depressive disorders and schizophrenia differ in the organization of motor activity both over short and long time periods. ${ }^{5,6}$

It has been thought that the time intervals between behavioural events usually occur randomly, following an exponential distribution and thus giving a straight line on a log-linear plot (probability vs. waiting time), meaning that very long interevent times should be very rare. There is now increasing evi- 
dence that many different types of human actions instead are characterized by bursts of activity separated by long periods with inactivity, examples of this being communication events, such as sending e-mails or making telephone calls. ${ }^{12}$ Such actions will follow a heavy-tail distribution, and on a log-log plot (probability vs. waiting time) this will appear as a straight line, suggestive of a power-law distribution.

With this as background, and using actigraphs to measure human activity, Nakamura et al. ${ }^{13}$ and Sano et al. ${ }^{14}$ have found that the distribution of active and inactive periods can be used to characterize the behavioural organization of patients with depression and schizophrenia, and that these distributions show differences compared to healthy controls. Furthermore, Nakamura et al., ${ }^{15}$ found that the organization of motor activity of mice displayed a similar pattern, such that the cumulative distribution of resting period durations seemed to obey a power-law distribution, and they also found that mice lacking clock genes differed from normal mice.

The aims of the present study has been to use these methods to reanalyze our actigraph registrations of depressed and schizophrenic patients in order to see if we can 1) replicate the findings of Nakamura et al. ${ }^{13}$ and Sano et al. ${ }^{14}$ and 2) to look for possible correlations between these findings and the altered variability and complexity parameters found in short- and long-term recordings from the same patients.

\section{METHODS}

\section{Ethics statement}

The study protocol was approved by the Norwegian Regional Medical Research Ethics Committee West. Written informed consent was obtained from all participants involved in the study.

\section{Subjects}

The study group consisted of 24 psychotic patients ( 3 women and 21 men), all with a diagnosis of schizophrenia, from an open ward for long-term patients (Knappentunet in Bergen) and 23 patients with mood disorders (10 women and 13 men), all currently depressed, five inpatients from an open psychiatric ward and 18 outpatients, all from the Haukeland University Hospital in Bergen), reported on in two previous papers. ${ }^{5,6}$ The control group consisted of 18 women and 11 men, average age $37.8 \pm 13.3$ years (mean $\pm S D$ ), range $21-66$, medical students $(n=5)$, patients without serious medical or psychiatric symptoms from a primary care office $(n=4)$ and employees from Knappentunet ( $n=20)$. None of the control subjects had a history of affective or psychotic symptoms.

\section{Psychiatric assessment}

All diagnostic assessments of the depressed patients were performed by one of the authors (OBF) using used a semistructured interview based on DSM-IV criteria ${ }^{16}$ for mood disorders. Depressive symptoms were assessed by the use of the Montgomery-Asberg Depression Rating Scale (MADRS) ${ }^{17}$

Diagnostic evaluations of the chronic psychotic patients were made by another of the authors (JØB) and a consensus diagnosis, based on DSM-IV criteria, was made after discussion of each case with OBF. Psychotic symptoms were evaluated by the use of the Brief Psychiatric Rating Scale (BPRS) ${ }^{18}$

The 23 depresssed patients fulfilled criteria for major depression according to DSM-IV. They had a mean age $42.8 \pm 11.0$ years. Fifteen had a major depressive disorder, one a bipolar I disorder and 7 bipolar II disorder. Their mean MADRS score at the start and end of registration were $22.7 \pm 4.8$ (range 13-29) and 20.0土4.7 (range 11-28), respectively.

The group of 24 patients with schizophrenia had mean age $47.4 \pm 11.1$ years (range 27-69 years). Their mean age at first hospitalization was $24.4 \pm 9.3$ years (range $10-52$ years). Eighteen had a paranoid form of schizophrenia. We were able to obtain BPRS scores in 19 of the 24 schizophrenic patients, with a mean of $51.5 \pm 9.5$ (range 34-68).

Eight of the depressed patients received no psychopharmacological treatment at the time of the study, of the rest $(n=15)$ all received either one $(n=13)$ or two $(n=2)$ antidepressants, five used lithium and one valproate. Five used antipsychotic drugs, mostly in small doses, and three used hypnotics or benzodiazepines. All the schizophrenic patients used antipsychotic drugs, 9 used clozapine, 8 used second generation drugs, 6 traditional antipsychotics, and two a combination of traditional and second generation drugs.

\section{Recording of motor activity}

Motor activity was monitored with an actigraph worn at the right wrist (Actiwatch, Cambridge Neurotechnology Ltd., England, UK). In the actigraph, activity is measured by using a piezoelectric accelerometer that is programmed to record the integration of intensity, amount and duration of movement in all directions. The sampling frequency is $32 \mathrm{~Hz}$ and movements over $0.05 \mathrm{~g}$ will be recorded. A corresponding voltage is produced and is stored as an activity count in the memory unit of the actigraph. The number of counts is proportional to the intensity of the movement. The right wrist was chosen to make the procedure more convenient for the participants, since most of them have their watches around the left wrist and it is cumbersome to have two such devices on the same arm. Previous studies have shown that there are only small differences between the right and left wrist. ${ }^{19,20}$ Total activity counts were recorded for one minute intervals for a continuous period of 
at least 12 days for all participants. Patients were instructed to remove the actigraphs when taking a bath/shower, and to record these time intervals. The recorded activity counts (0) in these sequences were then replaced with the mean for the whole recording period.

\section{Mathematical analyses}

Each recorded interval (one minute) was defined as either active or inactive, according to whether the activity count was above (or equal to) or below $10 \%$ of the mean for the whole recording period (17280 minutes) for that participant. An active or inactive period was defined as a continuous sequence of active or inactive intervals, and each recording was in this way divided into active or inactive periods with a length from one minute and upwards. For each recording the cumulative probability $(\mathrm{P})$ that an active or inactive period had a length of $\geq \mathrm{A}$ minutes was determined. For both active and inactive periods the following values of A were used: 1 to 5 with one minute intervals, 6 to 101 with 5 minute intervals, 151 to 451 with 50 minute intervals. For each participant the following numbers were calculated: mean activity count for the whole recording period, mean duration for active and inactive periods, and the longest active and inactive period. From the previous works of Nakamura et al. ${ }^{13}$ and Sano et al. ${ }^{14}$ we assumed we would find an approximately straight line on a log-log plot of $\mathrm{A}$ (minutes) versus $\mathrm{P}$ for inactive periods in both patient groups and controls, suggestive of a power law distribution, and a stretched exponential function for the active periods. However, in preliminary analyses of our data, we found that for low values of $\mathrm{A}$ ( $\leq 35 \mathrm{~min}$ for active sequences and $\leq 20$ min for inactive sequences) it seemed that a straight line best fitted the data on log-log plots. We therefore decided to calculate the slope of the line (the scaling exponent of a correspond- ing power function) that best fitted the data using the least squares method, using these values of A. The slope is negative, but in the table absolute values are given. We also calculated the number of active periods (expressed as \% of all the active periods) with a length (A) of 36 min or longer, and the number of inactive periods (\% of all the inactive periods) with a length (A) of $21 \mathrm{~min}$ or longer.

\section{Statistics}

One-way ANOVA was employed to evaluate differences between groups, with the p-value set at 0.05 , and post hos Bonferroni tests. Pearson's correlation coefficient was employed to evaluate correlations. SPSS version 18 was used was used for the statistical analyses.

\section{RESULTS}

The amount of time (mean $\pm \mathrm{SD}$ ) the actiwatch was removed from the arm was $1.0 \pm 0.9 \%$ of the total time for control subjects, $0.4 \pm 0.4 \%$ for the schizophrenic patients and $1.0 \pm 1.4 \%$ for the depressed patients. Compared to the controls both the schizophrenic and the depressed patients had lower mean activity scores (33\% and 28\% lower) (Table 1). The longest active period overall was $821 \mathrm{~min}$ (a control subject) and the longest inactive period was $830 \mathrm{~min}$ (a schizophrenic patient). There were no significant differences between the three groups in the mean length of longest active period, but the mean length of the longest inactive period was significantly higher in the schizophrenic patients compared to controls (135\% higher) (Table 1). The mean length of the active periods was significantly lower in the depressed patients compared to the controls (33\% lower) (Table 1), while the schizophrenic patients had a higher mean value for inactive period duration com-

Table 1. Actigraphic recordings of 12 days of motor activity (mean \pm SD)

\begin{tabular}{lcccc}
\hline & Controls $(\mathrm{n}=29)$ & Schizophrenia $(\mathrm{n}=24)$ & Depression $(\mathrm{n}=23)$ & $\mathrm{ANOVA}$ \\
\hline Activity count/min & $199 \pm 55$ & $134 \pm 49^{* * *}$ & $144 \pm 49^{* *}$ & $\mathrm{~F}(73,2)=12.306, \mathrm{p}<0.001$ \\
Active period duration & $9.6 \pm 2.0$ & $8.5 \pm 3.0^{\dagger \dagger}$ & $6.4 \pm 1.6^{* * *+\dagger}$ & $\mathrm{F}(73,2)=12.726, \mathrm{p}<0.001$ \\
Inactive period duration & $6.0 \pm 1.0$ & $7.8 \pm 2.3^{* * *+\dagger \dagger}$ & $5.9 \pm 1.3^{\dagger+\dagger}$ & $\mathrm{F}(73,2)=11.344, \mathrm{p}<0.001$ \\
Longest active sequence & $322 \pm 194$ & $249 \pm 158$ & $258 \pm 170$ & $\mathrm{~F}(73,2)=1.382, \mathrm{NS}$ \\
Longest inactive sequence & $89 \pm 29$ & $209 \pm 191^{* *}$ & $141 \pm 145$ & $\mathrm{~F}(73,2)=5.157, \mathrm{p}=0.008$ \\
Active sequences $\geq 36$ min (\%) & $7.0 \pm 2.2$ & $5.3 \pm 3.0^{* \dagger}$ & $3.5 \pm 1.8^{* * * \dagger}$ & $\mathrm{F}(73,2)=14.132, \mathrm{p}<0.001$ \\
Inactive sequences $\geq 21$ min (\%) & $7.2 \pm 2.2$ & $9.7 \pm 3.7^{* *+t \dagger}$ & $6.4 \pm 2.2^{+\dagger \dagger}$ & $\mathrm{F}(73,2)=9.253, \mathrm{p}<0.001$ \\
Scaling exponent & & & & $\mathrm{F}(73,2)=5.446, \mathrm{p}=0.006$ \\
\multicolumn{1}{c}{ Active periods } & $0.819 \pm 0.103$ & $0.766 \pm 0.129^{\dagger \dagger}$ & $0.880 \pm 0.125^{\dagger \dagger}$ & $\mathrm{F}(73,2)=13.778, \mathrm{p}<0.001$ \\
\hline Inactive periods & $0.709 \pm 0.108$ & $0.810 \pm 0.168^{\dagger}$ & $0.933 \pm 0.183^{* * *+}$ &
\end{tabular}

Durations are given in minutes. $\%$ denotes $\%$ of the total number of periods. Post hoc Bonferroni tests: ${ }^{*} \mathrm{p}<0.05$, schizophrenia or depression compared to the control group; ${ }^{* *} \mathrm{p}<0.01$, schizophrenia or depression compared to the control group; ${ }^{* * *} \mathrm{p}<0.001$, schizophrenia or depression compared to the control group; ${ }^{\dagger} \mathrm{p}<0.05$, schizophrenia compared to depression; ${ }^{\dagger \dagger} \mathrm{p}<0.01$, schizophrenia compared to depression; ${ }^{t+t} \mathrm{p}<0.001$, schizophrenia compared to depression 
pared to controls and the depressed patients (30\% higher than controls) (Table 1). The depressed patients also have a lower percentage of active periods with a duration of $36 \mathrm{~min}$ or more, compared to both the controls and the schizophrenic patients (50\% and 34\% lower) (Table 1). Concerning inactive periods with a duration of $21 \mathrm{~min}$ or more, the depressed patients do not differ from controls, but the schizophrenic patients have a higher percentage of such periods compared to both the controls and the depressed patients (24\% and 52\% higher) (Table 1).

In Figure 1 are shown the log-log plots of $\mathrm{P}$ vs. active periods with a duration $\leq 250 \mathrm{~min}$ for controls (Figure 1A), depressed (Figure 1B) and schizophrenic (Figure 1C) patients. From visual inspection of these figures it is obvious that, for the whole length of the curves, they do not follow straight lines. However, in Figure 2 the corresponding log-log plots of active periods with a duration $\leq 35 \mathrm{~min}$ for controls (Figure 2A), depressed (Figure 2B) and schizophrenic (Figure 2C) patients are
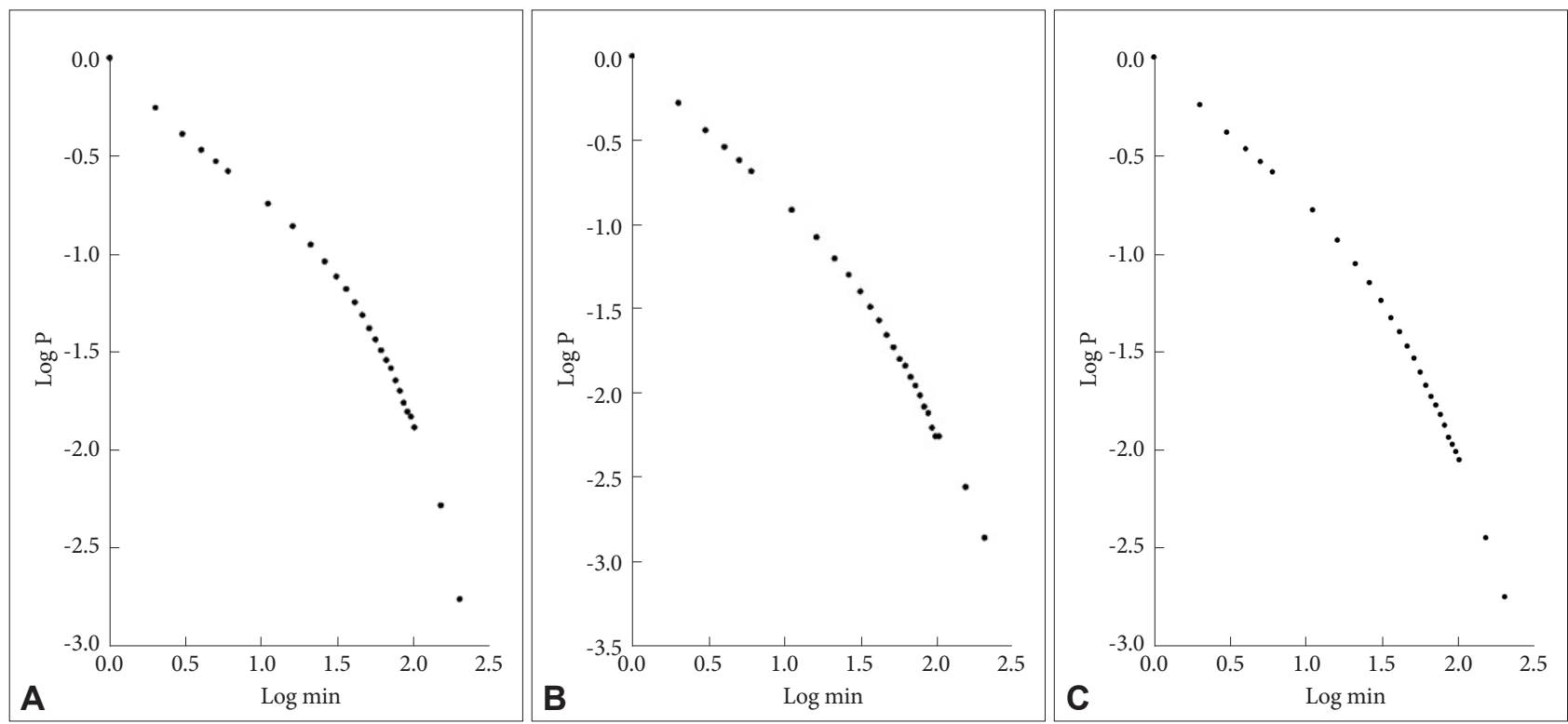

Figure 1. A: Log-log plots of cumulative probability $(P)$ vs. duration of active periods $(\leq 250 \mathrm{~min})$ for controls. B: Log-log plots of cumulative probability $(P)$ vs. duration of active periods $(\leq 250 \mathrm{~min})$ for depressed patients. C: Log-log plots of cumulative probability $(P)$ vs. duration of active periods ( $\leq 250 \mathrm{~min})$ for schizophrenic patients.
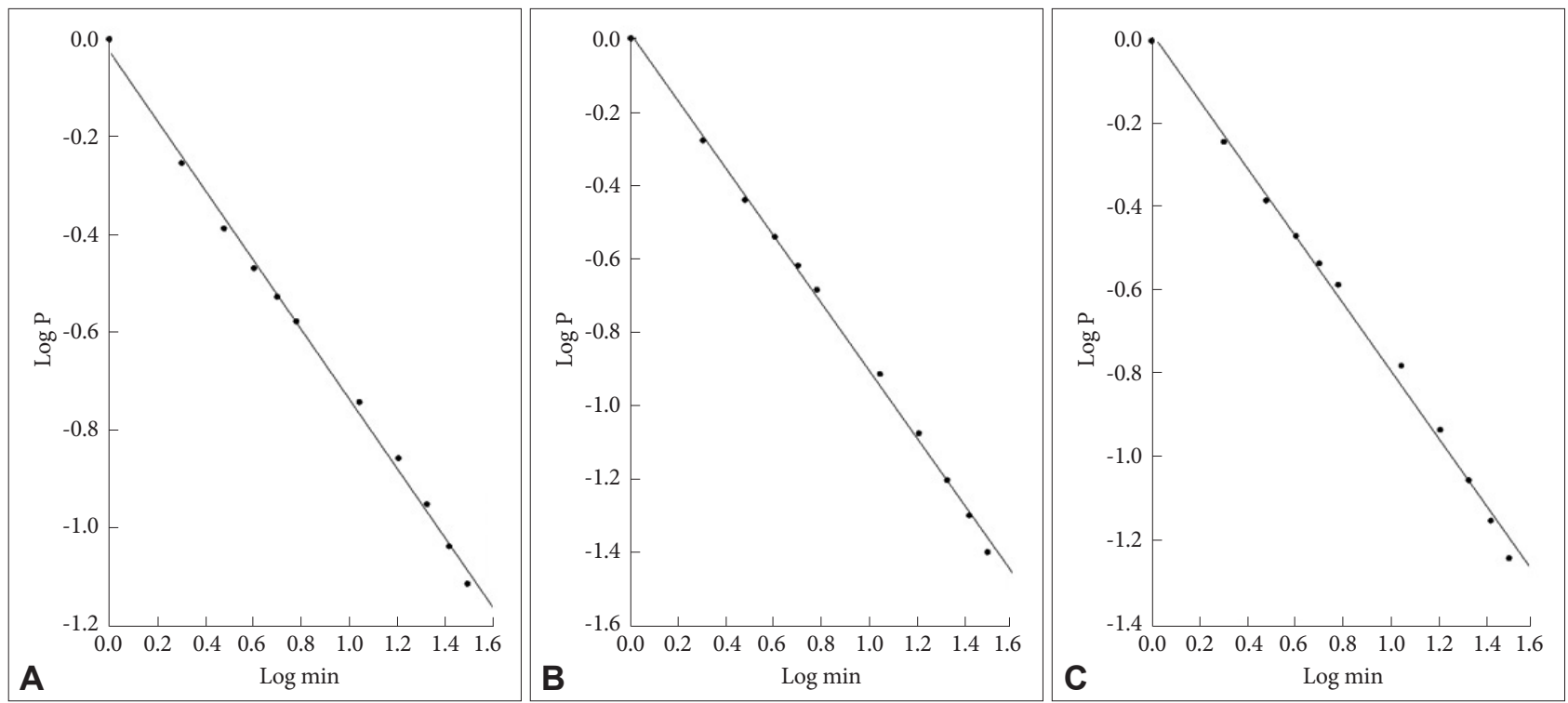

Figure 2. A: Log-log plots of cumulative probability $(P)$ vs. duration of active periods ( $\leq 35 \mathrm{~min}$ ) for controls. The straight line represents the linear regression line, using the least squares method. B: Log-log plots of cumulative probability $(P)$ vs. duration of active periods $(\leq 35$ min) for depressed patients. The straight line represents the linear regression line, using the least squares method. C: Log-log plots of cumulative probability $(P)$ vs. duration of active periods $(\leq 35 \mathrm{~min})$ for schizophrenic patients. The straight line represents the linear regression line, using the least squares method. 
shown, and all these three curves seem to be approximately straight lines, suggestive of power law functions. In Table 1 are shown the mean values for the slope of these lines, the value for the schizophrenic patients is lower than for controls, for the depressed patients it is higher, and the difference between the schizophrenic and depressed patients is statistically significant (the value for the depressed patients is $15 \%$ higher compared to the schizophrenic patients).

In Figure 3 are shown the log-log plots of $\mathrm{P}$ vs. inactive peri- ods with a duration $\leq 250$ min for controls (Figure 3A), depressed (Figure 3B) and schizophrenic (Figure 3C) patients. As for the active periods, visual inspections of these figures show that they do not follow straight lines. However, in Figure 4 the corresponding log-log plots for inactive periods with a duration $\leq 20 \mathrm{~min}$ for controls (Figure 4A), depressed (Figure 4B) and schizophrenic (Figure 4C) patients are shown, and all these three curves seem to be approximately straight lines. In Table 1 are shown the mean values for the slope of these lines, the value

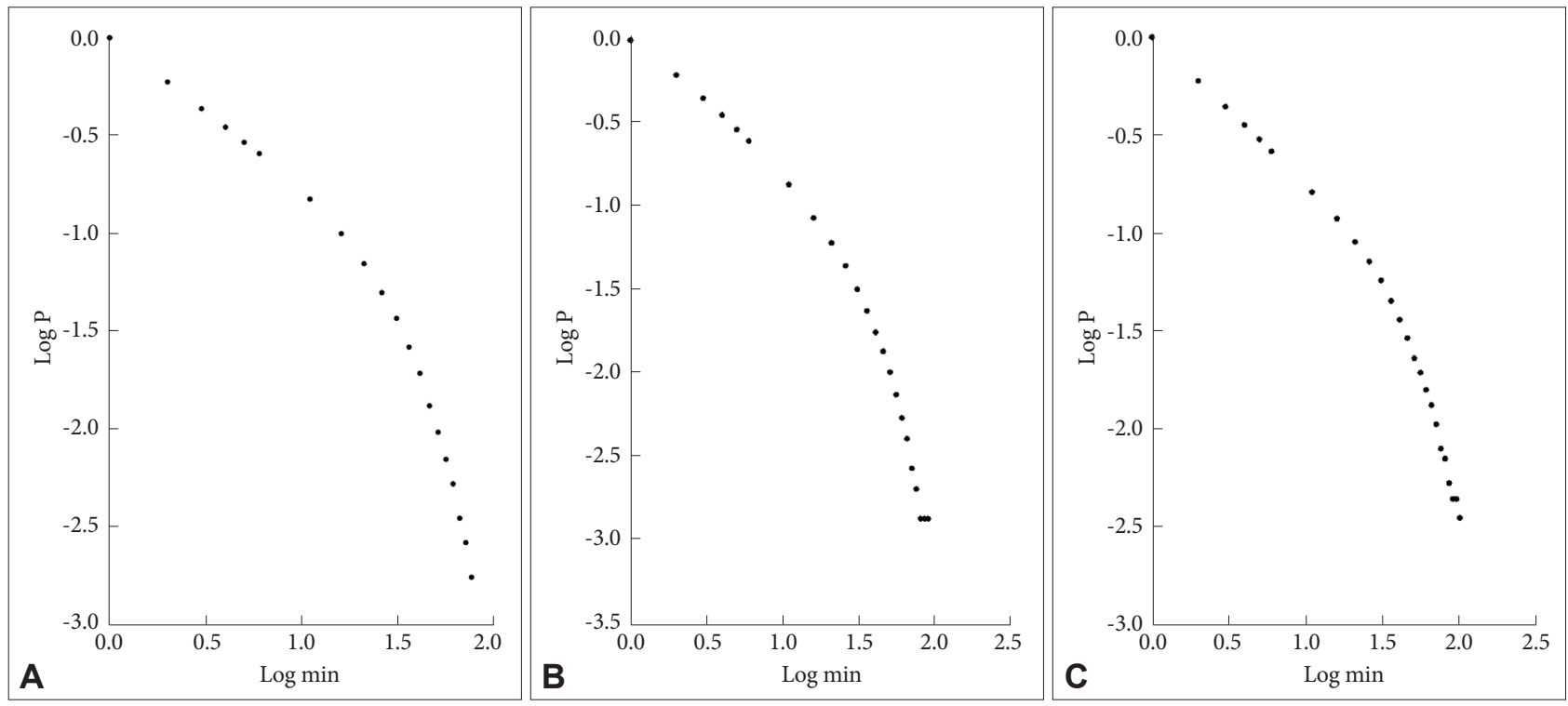

Figure 3. A: Log-log plots of cumulative probability $(P)$ vs. duration of inactive periods $(\leq 250 \mathrm{~min})$ for controls. B: Log-log plots of cumulative probability $(P)$ vs. duration of inactive periods $(\leq 250 \mathrm{~min})$ for depressed patients. C: Log-log plots of cumulative probability $(P)$ vs. duration of inactive periods ( $\leq 250 \mathrm{~min})$ for schizophrenic patients.
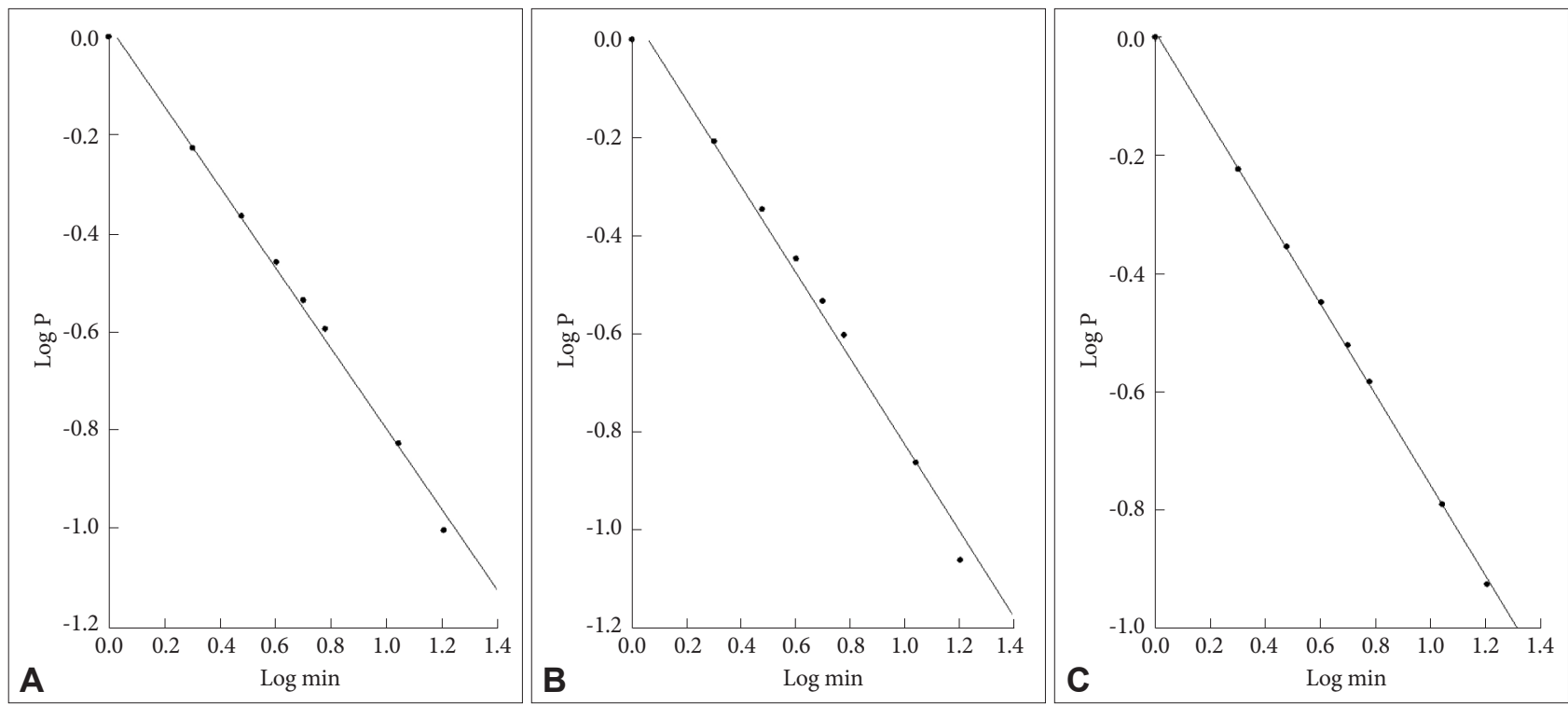

Figure 4. A: Log-log plots of cumulative probability $(P)$ vs. duration of inactive periods $(\leq 20 \mathrm{~min})$ for controls $(A)$. The straight line represents the linear regression line, using the least squares method. B: Log-log plots of cumulative probability $(P)$ vs. duration of inactive periods ( $\leq 20 \mathrm{~min}$ ) for depressed patients. The straight line represents the linear regression line, using the least squares method. C: Log-log plots of cumulative probability $(P)$ vs. duration of inactive periods ( $\leq 20 \mathrm{~min})$ for schizophrenic patients. The straight line represents the linear regression line, using the least squares method. 
for the controls is lowest, the schizophrenic patients have a higher value ( $14 \%$ higher compared to controls), and the depressed patients have the highest value (32\% higher compared to controls), statistically significant compared both to controls and to the schizophrenic patients.

When comparing males and females there were no significant differences for any of the parameters we used, when analyzed across the three groups (data not shown). Similarly there were no significant correlations for any of the parameters with age (data not shown).

In Table 2 are shown the correlations between some of the measures used in the present study. The most noteworthy findings are the very strong negative correlation between on one hand the scaling exponent of the inactive periods and the length of active periods $(-0.890)$, and on the other hand the scaling exponent of the active periods and the length of the inactive periods (-0.721). In addition the scaling exponent of the active periods is strongly negatively correlated to the amount of active periods with a length of $\geq 36 \mathrm{~min}$ $(-0.806)$, and the scaling exponent of the inactive periods is negatively correlated to the amount of inactive periods with a length of $\geq 21 \min (-0.472)$.
In Tables 3 and 4 are shown correlations between measures in the present study and measures we have used in previous studies on motor activity in the same groups of patients and controls. The two nonparametric variables in Table 3 interdaily stability (IS) and intradaily variability ${ }^{21}$ are used to analyse behavioural rhythms. ${ }^{22}$ The IS quantifies the invariability between the days, the strength of coupling of the rhythm to environmental factors. The IV indicates fragmentation of the rhythm, that is, the transitions between rest and activity. In the patients with schizophrenia, IS is increased and IV is reduced, reflecting a more structured behavioural pattern. ${ }^{5}$ The most noteworthy findings from Table 3 are the significant correlations of IS to all the six measures used in the present study, with positive correlations to the mean lengths of both active and inactive periods and to the amount of long active ( $\geq 36 \mathrm{~min})$ and inactive ( $\geq 21 \mathrm{~min}$ ) periods. In Table 4 are shown correlations between two measures used to characterize short term variability and complexity in recordings of 300 min durations; standard deviation (SD) and sample entropy, a measure of complexity of time series. ${ }^{6} \mathrm{SD}$ is negatively correlated to the length of active periods and to the amount of long active periods ( $\geq 36 \mathrm{~min})$ and positively

Table 2. Correlations between current measures

\begin{tabular}{|c|c|c|c|c|}
\hline & $\begin{array}{c}\text { Length of } \\
\text { active periods }\end{array}$ & $\begin{array}{c}\text { Length of } \\
\text { inactive periods }\end{array}$ & $\begin{array}{c}\text { Active periods } \\
\geq 36 \mathrm{~min}\end{array}$ & $\begin{array}{c}\text { Inactive periods } \\
\geq 21 \mathrm{~min}\end{array}$ \\
\hline Scaling exponents active periods & $-0.494^{* * *}$ & $-0.721^{* * *}$ & $-0.806^{* * *}$ & $-0.366^{*}$ \\
\hline Scaling exponents inactive periods & $-0.890^{* * *}$ & -0.220 & -0.144 & $-0.472^{* * *}$ \\
\hline
\end{tabular}

${ }^{*} \mathrm{p}<0.05,{ }^{* * *} \mathrm{p}<0.001$

Table 3. Correlations between current measures and rhythm analysis parameters

\begin{tabular}{lcc}
\hline & Interdaily stability & Intradaily variability \\
\hline Length of active periods & $0.318^{* *}$ & $-0.271^{*}$ \\
Length of inactive periods & $0.317^{* *}$ & -0.182 \\
Active sequences $\geq 36$ min (\%) & $0.262^{*}$ & -0.188 \\
Inactive sequences $\geq 21$ min (\%) & $0.360^{* *}$ & -0.196 \\
Scaling exponents active periods & $-0.403^{* * *}$ & 0.202 \\
Scaling exponents inactive periods & $-0.321^{* *}$ & $0.280^{*}$ \\
\hline
\end{tabular}

${ }^{*} \mathrm{p}<0.05,{ }^{* *} \mathrm{p}<0.01,{ }^{* * *} \mathrm{p}<0.001$

Table 4. Correlations between current measures and measures of variability and complexity

\begin{tabular}{lcc}
\hline & Standard deviation & Sample entropy \\
\hline Length of active periods & $-0.429^{* * *}$ & $0.324^{* *}$ \\
Length of inactive periods & -0.150 & $0.263^{*}$ \\
Active sequences $\geq 36$ min (\%) & $-0.412^{* * *}$ & $0.466^{* * *}$ \\
Inactive sequences $\geq 21$ min (\%) & -0.160 & $0.229^{*}$ \\
Scaling exponents active periods & 0.156 & -0.152 \\
Scaling exponents inactive periods & $0.419^{* * *}$ & $-0.228^{*}$ \\
\hline
\end{tabular}

${ }^{*} \mathrm{p}<0.05,{ }^{* *} \mathrm{p}<0.01,{ }^{* * *} \mathrm{p}<0.001$ 
correlated to the scaling exponent of inactive periods. Sample entropy is also correlated to the same measures, but the correlation is positive to the length of active periods and to the amount of long active periods ( $\geq 36 \mathrm{~min}$ ), and negative to the scaling exponent of inactive periods. Sample entropy is also positively correlated to the mean length of inactive periods and the amount of long inactive periods ( $\geq 21 \mathrm{~min}$ ), while SD is negatively (but not significantly) correlated also to these two measures.

\section{DISCUSSION}

The main finding of the present study is that patients with schizophrenia and depression have distinctly different profiles with regard to the distribution of active and inactive periods of motor activity. This adds to previously published results, with other mathematical methods, and suggests that there are important differences in control systems regulating motor behavior in these two major groups of psychiatric disorders.

In depressed patients, also using actigraph registrations, Nakamura et al. ${ }^{13}$ found that when examining the cumulative probability distribution $(\mathrm{P})$ of inactive periods, with lengths (A) from 2 to $200 \mathrm{~min}$, the relation of $\mathrm{P}$ to $\mathrm{A}$ on a log-log plot followed a straight line, suggestive of a power-law distribution, and the patients had a lower mean value for the scaling exponent compared to controls. This is the opposite of what we found, namely a higher value for the scaling exponent, and we have no clear explanation of this discrepancy. In agreement with Nakamura et al. ${ }^{13}$ we also found a straight line on a log$\log$ plot of the relation $\mathrm{P}$ to $\mathrm{A}$, but only for periods of $\mathrm{A} \leq 20$ $\mathrm{min}$, so obviously the distribution of the inactive periods, both in depressed patients and controls, is different in these two studies. The mean length of inactive periods of controls in the Nakamura study ${ }^{13}$ (inactive periods defined as non-zero activity counts) is quite similar to what we found (7.7 $\mathrm{min}$ vs. 6.0 $\mathrm{min})$, but the depressed patients in their study has a substantially higher value ( $15.6 \mathrm{~min}$ vs. $5.9 \mathrm{~min}$ ). However, it is important to note that the periods we have used to calculate the scaling exponents (having lengths $\leq 20 \mathrm{~min}$ ) comprise approximately $93 \%$ of all the inactive periods, and in studies from a wide range of different fields it not unusual that power-law behaviour only applies to a restricted range of parameter values. ${ }^{23}$ We have not performed a more rigorous study of whether our distributions follows a strict power-law function, ${ }^{23,24}$ but then our main purpose has not been to determine whether this is the case, but to look for differences between diagnostic groups.

In the study of Sano et al., ${ }^{14}$ with similar methods, for the cumulative probability distribution of inactive periods, schizophrenic patients were also found to have a significantly lower value for the scaling exponent compared to controls. We did not find any difference between schizophrenic patients and controls in our study, but in agreement with Sano et al. ${ }^{14}$ we found that the schizophrenic patients had a higher value for the mean length of inactive periods compared to controls. In agreement with this the schizophrenic patients also had a higher value for longest inactive period compared to controls (135\% higher). One difference between our patient groups is that we found a lower total activity count in the schizophrenic patients compared to controls, while Sano et al..$^{14}$ did not find any such difference.

For active periods we found that the cumulative probability distribution $(\mathrm{P})$ of these periods $(\mathrm{A})$, using lengths $(\mathrm{A})$ from 1 to $35 \mathrm{~min}$, followed a straight line, when plotting $\mathrm{P}$ vs. A on a log-log plot. Nakamura et al. ${ }^{13}$ and Sano et al. ${ }^{14}$ did not find such a relationship, but instead a stretched exponential function, when using the whole range of lengths. We cannot therefore directly compare our results concerning scaling exponents, but while we found no significant differences between any of the patient groups and controls, we found that the depressed patients had a significantly higher value for the scaling exponent compared to the schizophrenic patients. Nakamura et al. ${ }^{13}$ did not find any differences between depressed patients and controls concerning parameter values for the stretched exponential functions, but Sano et al. ${ }^{14}$ found that the schizophrenic patients were different from controls. Concerning active period durations we did not find any difference between schizophrenic patients and controls, in agreement with Sano et al., ${ }^{14}$ but the depressed patients in our study had a significantly lower value, compared both to controls and to schizophrenic patients.

We also looked at the number of active and inactive periods not employed for the calculation of the scaling exponents, that is active periods with a duration equal to or longer than $36 \mathrm{~min}$ and inactive periods with a duration equal to or longer than 21 $\min$. This revealed a pattern that discriminated even better between schizophrenic and depressed patients than the use of scaling exponents. The percentage of these long active periods in relation to the total number of active periods was significantly lower in the depressed patients compared to both controls and to the schizophrenic patients. For the long inactive periods the percentage was higher in the schizophrenic patients compared to both controls and depressed patients. Therefore, even though the total activity counts are similar in the two patient groups, the depressed patients are characterized by fewer long activity periods, and the schizophrenic patients by a higher number of long inactive periods. These relationships are in reasonable agreement with clinical experience, and easier to grasp than the results from the calculation of scaling exponents.

The correlations presented in Table 2 underscore the relationships between the calculation of scaling exponents, duration of active and inactive periods and number of long active and inactive periods. These relationships are clearly interrelat- 
ed, reflecting common mechanisms that regulate the transition between activity and rest.

There are moderate, but significant, correlations with parameters used in the rhythm analysis of van Someren, ${ }^{22}$ particularly interdaily stability (Table 3 ). The correlations are positive with mean lengths of active and inactive periods and number of long active and inactive periods, which is intuitively reasonable, since higher values of all these four measures presumably will tend to give a more stable rhythm. The correlations with the scaling exponents are negative, which again is reasonable given the results form Table 2 , showing negative correlations between the scaling exponents and the other four measures.

In our previous paper on mathematical analyses of motor activity time series, we reported a strong negative correlation between sample entropy values and SD. ${ }^{6}$ The correlations between sample entropy and SD, taken from analyses of $300 \mathrm{~min}$ sequences in the same group of patients, and scaling exponents, duration of active and inactive periods and number of long active and inactive periods, show moderate correlations, which are not easy to interpret, but probably reflect common mechanisms in these aspects of motor activity regulation. However, it is interesting to note that when sample entropy shows a negative correlation, the correlation with $\mathrm{SD}$ is positive, and the other way around.

Sano et al. ${ }^{14}$ found that the parameter $\beta$ of the stretched exponential function for active periods increased with age in schizophrenic patients. We did not find any correlations with age for any of our measures either in the whole study group or in the group of schizophrenic patients separately, neither did we find any correlation with scores on the Montgomery-Asberg Depression Rating Scale in the depressed patients or on the score on the Brief Psychiatric Rating Scale sin the schizophrenic patients (data not shown). However, we did not have patients with a wide range of severity, mainly moderately depressed outpatients and chronically ill, institutionalized schizophrenic patients. It would clearly have been an advantage to have a more heterogenous group in order to look at a possible relationship with severity. This also makes it difficult to know if our findings can be generalized to other groups of depressed and schizophrenic patients.

Actigraphy has been useful in studies on sleep, ${ }^{25}$ and it is possible that mathematical analyses of actigraph registrations from periods with continuous motor activity ${ }^{6,26}$ combined with analyses of active and inactive periods during longer registration periods may give a biological "signature" that can be useful for diagnostic purposes. It is obviously important if clinical impression of altered behavior can be supplanted with objective registrations of motor behavior. It will also be interesting to see if any of these measures can be used to predict effect of treatment, or be altered as the result of treatment. In the present study we used 12 days of registration, in the study of Nakamura et al..$^{13}$ seven days were used, and in Sano et al. ${ }^{14}$ five days. However, to make this more applicable to clinical practice it would be useful to see if analyses of active and inactive periods can be reliably applied to shorter to time periods, such as one or two days.

In addition, new technological developments are adding exciting possibilities for future studies, including use of smartphones in combination with activity measurements. ${ }^{27}$

Treatment with psychotropic medication may of course have influenced our results, perhaps foremost the findings from the schizophrenic patients, but it is difficult to separate such an influence from genuine biological differences between the groups. We have previously reported that patients treated with clozapine differed from schizophrenic patients treated with other antipsychotics, ${ }^{5}$ but in the present study there were no significant differences between patients treated with clozapine and the other schizophrenic patients.

The gender distribution is different in the three groups and this could be a possible confounding factor. However, we did not find differences between genders for any of the analyzed parameters.

The mean age of the schizophrenic patients is higher than the other two groups, and this may also be a relevant confounding factor. However, again we did not find any significant correlations between age and any of the analyzed parameters.

We have not made a separate analysis of sleep parameters, and sleep may be altered both in depressed ${ }^{28}$ and schizophrenic patients. ${ }^{29}$ This may have influenced our findings, but on the other hand it would be very difficult to disentangle such effects from other effects on rest and activity rhythms. The controls in our study were employed and working, or students, while the patients were not. This is a potential source of bias that is difficult to evaluate. It would also have been desirable to have larger study groups and longer observation time, but this was not possible to accomplish with the resources we had available.

Participants were asked to remove the actigraphs while taking a bath or showering, but this comprises only short time periods, and is unlikely to have biased the results.

Diagnoses were assessed non-blind, but the actigraphic registrations and subsequent mathematical analyses did not require subjective evaluations.

\section{Conclusions}

Bursts of activity are shorter in duration in depressed patients, and there are fewer long active periods, while periods of inactivity are of longer duration in schizophrenic patients and there is a higher number of long inactive periods. Calculation of scaling exponents shows that the depressed patients are more different from controls than schizophrenic patients, both 
for active and inactive periods. Overall the results give the impression that the distribution of active and inactive periods is more disturbed in depressed compared to schizophrenic patients. In this paper, we have thus presented findings highlighting a difference between depression and schizophrenia. These diseases have long been regarded to be categorically distinct. However, we submit the methodology utilized may have widespread application in addressing important and in some instances controversial matters of importance to psychiatric nosology.

\section{Acknowledgments}

We want to thank Erlend Fasmer for making the programs for extracting the active and inactive sequences from the actigraph recordings.

\section{REFERENCES}

1. Jung SH, Kim WH, Choi HJ, Kang MH, Lee JS, Bae JN, et al. Factors affecting treatment discontinuation and treatment outcome in patients with schizophrenia in Korea: 10-year follow-up study. Psychiatry Investig 2011;8:22-29.

2. Kim TS, Jeong SH, Kim JB, Lee MS, Kim JM, Yim HW, et al. The clinical research center for depression study: baseline characteristics of a korean long-term hospital-based observational collaborative prospective cohort study. Psychiatry Investig 2011;8:1-8.

3. Jeong HG, Ko YH, Han C, Kim YK, Joe SH. Distinguishing quantitative electroencephalogram findings between adjustment disorder and major depressive disorder. Psychiatry Investig 2013;10:62-68.

4. Haavik J, Halmoy A, Lundervold AJ, Fasmer OB. Clinical assessment and diagnosis of adults with attention-deficit/hyperactivity disorder. Expert Rev Neurother 2010;10:1569-1580.

5. Berle JO, Hauge ER, Oedegaard KJ, Holsten F, Fasmer OB. Actigraphic registration of motor activity reveals a more structured behavioural pattern in schizophrenia than in major depression. BMC Res Notes 2010; 3:149.

6. Hauge ER, Berle JO, Oedegaard KJ, Holsten F, Fasmer OB. Nonlinear analysis of motor activity shows differences between schizophrenia and depression: a study using Fourier analysis and sample entropy. PLoS One 2011;6:e16291.

7. Fink M. Rediscovering catatonia: the biography of a treatable syndrome. Acta Psychiatr Scand Suppl 2013;(441):1-47.

8. Walther S, Horn H, Razavi N, Koschorke P, Muller TJ, Strik W. Quantitative motor activity differentiates schizophrenia subtypes. Neuropsychobiology 2009;60:80-86.

9. Dane AV, Schachar RJ, Tannock R. Does actigraphy differentiate ADHD subtypes in a clinical research setting? J Am Acad Child Adolesc Psychiatry 2000;39:752-760.

10. Bussmann JBJ, Ebner-Priemer UW, Fahrenberg J. Ambulatory activity monitoring: Progress in measurement of activity, posture, and specific motion patterns in daily life. Eur Psychol 2009;14:142-152.

11. Burton C, McKinstry B, Szentagotai Tatar A, Serrano-Blanco A, Pagliari C, Wolters M. Activity monitoring in patients with depression: a systematic review. J Affect Disord 2013;145:21-28.
12. Barabasi AL. The origin of bursts and heavy tails in human dynamics. Nature 2005;435:207-211.

13. Nakamura T, Kiyono K, Yoshiuchi K, Nakahara R, Struzik ZR, Yamamoto Y. Universal scaling law in human behavioral organization. Phys Rev Lett 2007;99:138103.

14. Sano W, Nakamura T, Yoshiuchi K, Kitajima T, Tsuchiya A, Esaki Y, et al. Enhanced persistency of resting and active periods of locomotor activity in schizophrenia. PLoS One 2012;7:e43539.

15. Nakamura T, Takumi T, Takano A, Aoyagi N, Yoshiuchi K, Struzik ZR, et al. Of mice and men--universality and breakdown of behavioral organization. PLoS One 2008;3:e2050.

16. American Psychiatric Association. Diagnostic and Statistical Manual of Mental Disorders, 4th Edition - Text Revision. Washington DC: American Psychiatric Association; 2000.

17. Montgomery SA, Asberg M. A new depression scale designed to be sensitive to change. Br J Psychiatry 1979;134:382-389.

18. Overall JE, Gorham DR. The Brief Psychiatric Rating Scale. Psychological Reports 1962;10:799-812.

19. Van Hilten JJ, Middelkoop HA, Kuiper SI, Kramer CG, Roos RA. Where to record motor activity: an evaluation of commonly used sites of placement for activity monitors. Electroencephalogr Clin Neurophysiol 1993; 89:359-362.

20. Littner M, Hirshkowitz M, Davila D, Anderson WM, Kushida CA, Woodson BT, et al. Practice parameters for the use of auto-titrating continuous positive airway pressure devices for titrating pressures and treating adult patients with obstructive sleep apnea syndrome. An American Academy of Sleep Medicine report. Sleep 2002;25:143-147.

21. Goldberger AL, Amaral LA, Glass L, Hausdorff JM, Ivanov PC, Mark RG, et al. PhysioBank, PhysioToolkit, and PhysioNet: components of a new research resource for complex physiologic signals. Circulation 2000; 101:E215-E220.

22. Van Someren EJ, Swaab DF, Colenda CC, Cohen W, McCall WV, Rosenquist PB. Bright light therapy: improved sensitivity to its effects on rest-activity rhythms in Alzheimer patients by application of nonparametric methods. Chronobiol Int 1999;16:505-518.

23. Clauset A, Shalizi CR, Newman MEJ. Power-law distributions in empirical data. SIAM Rev 2009;51:661-703.

24. Kawabata M, Ueno T, Tomita J, Kawatani J, Tomoda A, Kume S, et al. Temporal organization of rest defined by actigraphy data in healthy and childhood chronic fatigue syndrome children. BMC Psychiatry 2013; 13:281.

25. Ancoli-Israel S, Cole R, Alessi C, Chambers M, Moorcroft W, Pollak CP. The role of actigraphy in the study of sleep and circadian rhythms. Sleep 2003;26:342-392.

26. Krane-Gartiser K, Henriksen TE, Morken G, Vaaler A, Fasmer OB. Actigraphic assessment of motor activity in acutely admitted inpatients with bipolar disorder. PLoS One 2014;9:e89574.

27. Hurvitz PM, Moudon AV, Kang B, Saelens BE, Duncan GE. Emerging technologies for assessing physical activity behaviors in space and time. Front Public Health 2014;2:2.

28. Dridi D, Zouiten A, Mansour HB. Depression: chronophysiology and chronotherapy. Biol Rhythm Res 2014;45:77-91.

29. Monti JM, BaHammam AS, Pandi-Perumal SR, Bromundt V, Spence DW, Cardinali DP, et al. Sleep and circadian rhythm dysregulation in schizophrenia. Prog Neuropsychopharmacol Biol Psychiatry 2013;43: 209-216. 